\title{
Practical usage of computer-supported outbreak detection in five European countries
}

A Hulth (anette.hulth@smi.se) ${ }^{1}$, N Andrews², S Ethelberg 3 , J Dreesman4, D Faensen ${ }^{5,6}$, W van Pelt7, J Schnitzler5,8

1. Swedish Institute for Infectious Disease Control, Stockholm, Sweden

2. Health Protection Agency, London, United Kingdom

3. Statens Serum Institut, Copenhagen, Denmark

4. Governmental Institute of Public Health of Lower Saxony, Hannover, Germany

5. Robert Koch Institute, Berlin, Germany (affiliation where the work was performed)

6. Current affiliation: European Centre for Disease Prevention and Control, Stockholm, Sweden

7. National Institute for Public Health and the Environment (RIVM), Bilthoven, the Netherlands

8. Current affiliation: World Health Organization, Geneva, Switzerland

Hulth A, Andrews N, Ethelberg S, Dreesman J, Faensen D, van Pelt W, Schnitzler J. Practical usage of computer-supported outbreak detection in five European countries. Euro Surveill. 2010;15(36):pii=19658. Available online: http://www.eurosurveillance.org/ViewArticle.aspx?Articleld=19658

This paper discusses computer-supported outbreak detection using routine surveillance data, as implemented at six institutes for infectious disease control in five European countries. We give an overview of the systems used at the Statens Serum Institut (Denmark), Health Protection Agency (England, Wales and Northern Ireland), Robert Koch Institute (Germany), Governmental Institute of Public Health of Lower Saxony (Germany), National Institute for Public Health and the Environment (the Netherlands) and Swedish Institute for Infectious Disease Control (Sweden). Despite the usefulness of the algorithms or the outbreak detection procedure itself, all institutes have experienced certain limitations of the systems. The paper therefore concludes with a list of recommendations for institutes planning to introduce computersupported outbreak detection, based on experiences on the practical usage of the systems. This list - which concerns usability, standard operating procedures and evaluation - might also inspire improvements of systems in use today.

\section{Introduction}

Over the past decade, a number of institutes for infectious disease control throughout Europe have gained experience of systems for computer-supported outbreak detection. There are several reasons for introducing such systems to complement the daily surveillance already performed, mainly: (i) to detect outbreaks earlier, (ii) to detect outbreaks that would probably not have been detected otherwise, and (iii) to highlight potential problematic increases in incidence of a disease in the pre-outbreak phase.

Outbreak detection starts with the detection of an aberrant number of reported cases (suspected or confirmed) of a particular disease in a given time and space. Computer programs are used to compare the observed number of cases with expected values. When an increase is detected, the computer program raises an alert (the signal). Next, an expert (for example, an epidemiologist) assesses the public health relevance of the aberration, to determine if further investigation is warranted. Such investigations - which may involve a number of people at international, national and local level - are aimed at confirming whether there is an outbreak or not. If an outbreak is confirmed, further investigations will follow, where, for example, the magnitude of the outbreak is assessed, the source is traced and control measures are suggested. The task of the system is thus to warn of possible outbreaks. The process is outlined in the Figure.

Many algorithms can be used to detect deviations in infectious disease data, ranging from simple fixed thresholds to the application of complex statistical methods taking, for example, historical data into account (for reviews, see, for example, [1] or [2]). These algorithms can be applied to both laboratory data and clinical diagnoses as well as to syndromic surveillance data. Algorithms can be used for both geospatial and time series data. Considerable research has been carried out to improve these algorithms, that is, to increase specificity while reducing noise. To our knowledge, there are, however, no documented best practices on how to deal with the detected signals.

As part of a Swedish national project on computer-supported outbreak detection, the Swedish Institute for Infectious Disease Control contacted all focal points in the 27 countries that had participated in the former Basic Surveillance Network (BSN), in September 2006. (BSN was a European network for sharing national case-based reports on infectious diseases [3], which constitutes the basis for the current European surveillance system (TESSy) [4] maintained by the European Centre for Disease Prevention and Control (ECDC).) The country contacts were asked if their institute was using any form of electronic outbreak detection or had any information on the issue. A total of 19 replies were 
received. National institutes in the following countries had experience to share with the Swedish institute: Denmark; England, Wales and Northern Ireland; Germany; the Netherlands; and Norway. These countries were subsequently sent a more detailed questionnaire and a dialogue was initiated. One result of this dialogue was a workshop on presenting and interpreting automatic outbreak detection signals, held at the Robert Koch Institute in Berlin, Germany, in May 2007, with participants from the six countries, along with representatives from the World Health Organization and ECDC. In November 2008, a second workshop was held, at which it was agreed that the institutes with computer-supported systems in place and represented at the meeting should share their experiences with institutes planning to introduce such systems.

In this paper, we describe how six surveillance institutes in five European countries have implemented computer-supported outbreak detection in their routine surveillance, giving an overview of how they are currently used, along with the lessons learnt. We also provide some recommendations for institutes that plan to introduce similar systems. The paper does not describe infectious disease control in the contributing countries, nor is the description of the implemented computer-supported systems exhaustive.

\section{Country experiences}

The countries that describe their experiences of computer-supported outbreak detection in this article vary in population size, ranging from a small country such as Denmark, with about 5.5 million people, to a large country such as Germany, with more than 82 million. Although infectious disease control is structured differently in each of the five countries, there are a number of common experiences in using the systems in daily work.

The authors describe the computer-supported outbreak detection system of their institution either as person in charge of the system, as the main user, or in collaboration with the users of the system. The perspective is that of the user of the system: it does not focus on the performance of the underlying algorithms. Although figures showing, for example, the sensitivity and positive predictive value of a particular algorithm applied to data collected in a particular country for a particular disease will reveal some information about its performance, there are many other aspects that are even more important, which are addressed in this paper.

\section{Denmark}

In Denmark, each week all clinical laboratories are required to report to a national database personidentifiable information on cases found positive for pathogenic gastrointestinal bacteria. Since 2001, an automated outbreak detection system based on these data has been in use at the Statens Serum Institut, generally running once a week. The system is an implementation, made in the statistical software SAS, of the algorithm described by Farrington et al. [5]. This algorithm uses Poisson regression on weekly counts of cases positive for each bacterial agent. Both national data and data from each laboratory's uptake area are analysed and the possibility of an outbreak is expressed on a scale from one to 10 by the system. Results are evaluated by an epidemiologist and signals deemed relevant are communicated by email to the appropriate investigators or discussed at weekly national inter-institutional outbreak meetings. In addition, surveillance and outbreak algorithm results for the most frequent bacterial agents have been published as maps, graphs and tables on a designated public website (http://www.germ.dk) on a weekly basis. Molecular subtyping data are not part of the algorithm. The algorithm has proven to be a useful surveillance tool, particularly for salmonella infections [6]. It has helped detect several outbreaks that might otherwise not have been noted at the time, both nonpoint source (diffuse) outbreaks of disease due to rare serotypes and local outbreaks resulting from frequent serotypes.

\section{FIGURE}

Process of computer-supported outbreak detection, involving both computerised and manual elements

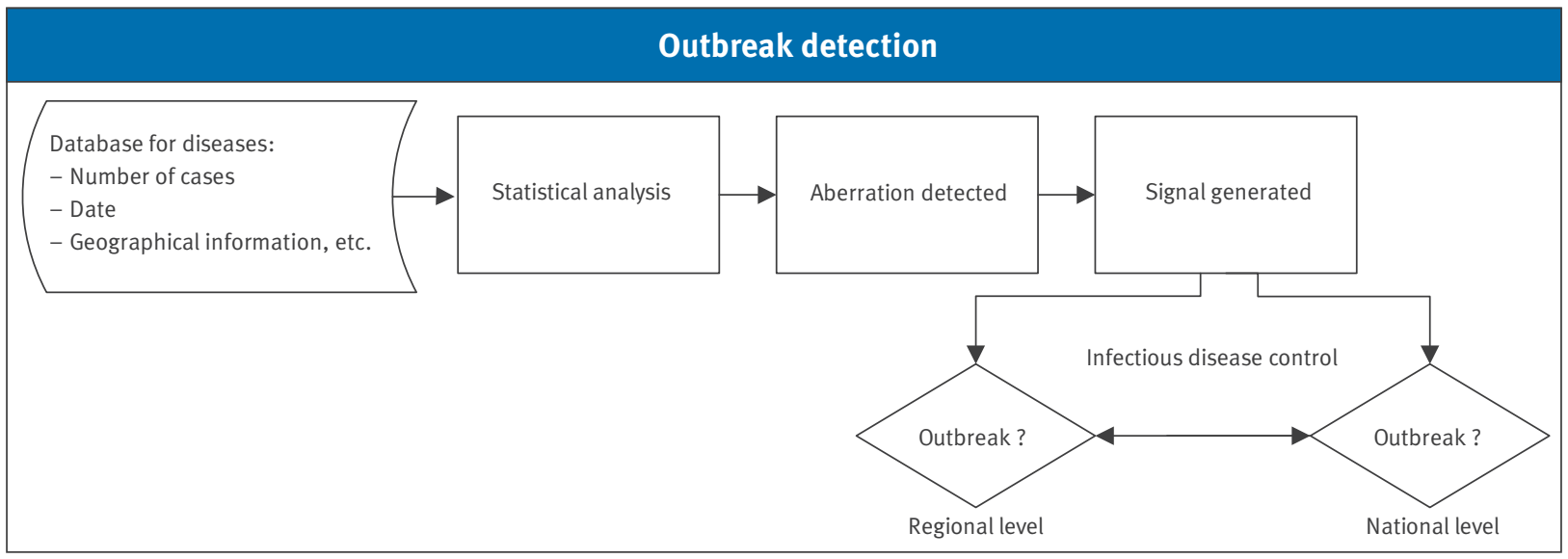


England, Wales and Northern Ireland

In England, Wales and Northern Ireland all clinical laboratories are asked to electronically submit details of all organisms isolated to a laboratory database at the Centre for Infections of the Health Protection Agency (HPA). The information sent by the clinical laboratories will have come from patients within hospital departments or those attending general practices. Since 1993 an automated algorithm, developed by Farrington et al. [5], has been used weekly to detect possible outbreaks by comparing the current week's total reports for each organism with a threshold calculated using Poisson regression on the past five years' data. Analyses are run using all regions combined and also within each of 11 regions, producing lists of all organisms, ranked according to the level of exceedance above the threshold. For organisms with an exceedance (typically five to 20 organisms per week), plots are also produced showing the time series and the distribution of cases by age group and region or district along with an indication if this differs significantly from the past age group or regional distribution of cases. Results are posted on the HPA intranet and are also emailed to national and regional epidemiologists who further investigate the exceedances where necessary and initiate an outbreak investigation if appropriate. A weekly teleconference, based in the Centre for Infections, is held with national and regional epidemiologists to discuss any signals. The algorithm is currently being updated to allow data to be aggregated according to the date the clinical specimen was taken rather than date of receipt of the case report at the Centre for Infections. This may enable more rapid detection of outbreaks and reduce false signals, but it does require allowance for reporting delays in the model. The algorithm has enabled detection of outbreaks (particular salmonella) not otherwise identified, but the number of false signals and delays in reporting have limited its usefulness.

\section{Germany - national level}

In Germany, approximately 60 pathogens and health issues are reported by laboratories, general practitioners or other entities to the local health authorities $[7,8]$. Detailed information about cases is entered into a decentralised database, anonymised and transferred via the state health department at the regional level to the national level - the Robert Koch Institute (RKI). The RKI runs automated outbreak detection on the case reports [9], using a slightly modified version of the algorithm described by Stroup et al. [10]. By applying the algorithm to subsets of the data, such as certain regions (Bundesland, county), age groups, sex, countries of infection, etc. it is possible to detect outbreaks in a population group even if the excess cases would be undetectable when looking at the whole population. Cases can be linked to electronic outbreak reports at the different administrative levels [8].

The automated outbreak detection runs weekly. The detected aberrations are recorded on Microsoft Excel spreadsheets, including information on the particular subset of the data that led to the signal. A trained administrative clerk screens these signals and notifies the epidemiologist in charge when a signal subjectively seems to require further action. This decision is based on, for example, the disease, strength of signal and whether or not it is related to an outbreak that has already been detected at the local or regional level.

In 2002, the RKI tried to visualise the alerts, with a graphical output. A system presented the cases and incidence for each disease at each administrative level in charts and maps, including the possibility of showing the place of residence of the affected cases [11]. The main advantage of that system was that the user could label a signal as handled, avoiding repeated presentation of the same signal when running the algorithms daily. Unfortunately, the implementation of the surveillance system's front end did not easily allow for such an extension and this tool was therefore developed as an independent application. This lack of integration negatively influenced the usability and the tool was never incorporated into the regular surveillance.

\section{Germany - Lower Saxony}

At the governmental institute of public health in the German Land of Lower Saxony (NLGA), a system for automated outbreak detection has been developed with freeware tools. The starting point is case counts aggregated by disease (for salmonellosis, also by serotype of the causative agent), week of notification and 46 administrative districts. The data are exported weekly from the case database at the NLGA [7]. The following statistical methods (and corresponding software) are applied: detection of clusters in time by the method of Stroup et al. [10] and the method developed by Farrington et al. [5], as implemented in the R package surveillance [12], as well as detection of spatial clusters by SaTScan spatial scan statistics [13]. Data are also visualised on a website through time series charts using R software and maps (EpiMap) [14]. Validation of the signals since 2002 suggests that attention should be focused on highly significant signals (p<0.01). The results vary widely between diseases due to their different epidemiological characteristics. Spatial clustersignals are frequently caused by diagnostic effects, for instance, by a tuberculosis screening programme in an immigration centre [15] or by specific awareness for Cryptosporidium parvum in a regional laboratory [16]. The methods are primarily valuable for noticing case clusters at an early stage. However, the initial suspicion or even detection of the clusters has often already occurred elsewhere - for example, at a local public health department or in a laboratory. Besides cluster identification, the statistically justified cluster signals have been proven to be helpful for communication purposes and decision support.

\section{The Netherlands}

In the Netherlands, for notifiable diseases (except salmonella and campylobacter infections) the simple model of Stroup et al. [10] has been used at the National Institute for Public Health and the Environment (RIVM) 
since 1998. Laboratory surveillance, however, is voluntary and based on a sentinel of clinical laboratories that has been difficult to sustain. At RIVM, the algorithm developed by Farrington et al. [5] was implemented and in use from 2002 to 2006 for 34 pathogens [17], and from 1996 to date for more than 700 salmonella serotypes and phagetypes on a weekly basis. Observed, expected and tolerance levels are presented as time series that can be visually inspected retrospectively; observed frequencies are flagged if above the defined level of tolerance. A one- and a four-week window is used and a weekly window is run day by day, to improve sensitivity [17]. For salmonellosis, a website is available on the RIVM intranet, showing the period above tolerance levels and if cases are significantly clustered in space or demographically deviating from expected results. Maps are automatically generated each week for significant clusters. Out of hundreds of pathogens and serotypes analysed, the system draws attention to those signals that need further investigation and aids in the first steps of signal verification. Results are evaluated by an epidemiologist and signals deemed relevant are communicated by email and discussed weekly together with other signals, to decide upon further action [18]. Attention has been drawn to numerous small and large outbreaks in the past 10 years of using this system of algorithms and presentation of underlying information [19].

\section{Sweden}

The Swedish Institute for Infectious Disease Control has implemented a framework for computer-supported outbreak detection, called Computer Assisted Search for Epidemics (CASE) [20]. The source code for the framework is available as open source, licensed under the General Public License GPLv3 [21]. There is no limit set to the number of statistical algorithms that the CASE framework can support, and one or more algorithms can be applied to each disease. In addition, the parameter settings for each algorithm can be different for different diseases, even for different types if required. When an aberration is detected, an email is sent to the people listed for the particular disease, such as the epidemiologist in charge of that disease. The database behind CASE is populated with disease, disease agent type (when available) and regional information (county code), and the date when a case was first entered in the database. Two SaTScan algorithms $[13,22]$ are fully integrated in the system, as are the algorithm described by Farrington et al. [5], as implemented in [12], and OutbreakP, which is used to investigate if an increase in the number of cases is more than expected, thus implying a potential outbreak [23]. In addition, a simple threshold can be set, where the number of reported cases is not to exceed a manually predefined value. Specified parts of the output generated by the algorithms are automatically extracted and processed further - for example, a signal is conveyed to the person responsible for the surveillance of the disease in question only if it occurred during the two preceding weeks. The system is implemented for all 62 notifiable diseases in Sweden. The algorithms are still being fine-tuned to suit the diseases, in order to find a reasonable balance between false alarms and not missing true outbreaks.

\section{Recommendations}

Drawing on everyday experience with computersupported outbreak detection from all the institutes represented in this paper, we present a number of recommendations. These recommendations, summarised in the checklist (Box), should be valuable not only to countries wishing to implement their own system, but also to those that already have such a system in place. On the basis of practical experience, we consider that complying with these recommendations is a prerequisite for an optimally functioning computer-supported outbreak detection system.

\section{Box}

Checklist for a computer-supported outbreak detection system

- $\quad$ Signals and alerts are presented in a way that works well for the receivers.

- The output is user friendly, preferably in a graphical format (maps, epidemic curves etc.).

- $\quad$ People in charge of the surveillance of a particular disease can obtain signals for only that disease.

- $\quad$ The system is tightly integrated with the database, giving easy access to the case reports that contributed to the signals.

- $\quad$ Feedback from the receivers of the alerts is continuously incorporated into the system.

- $\quad$ Feedback to the public health workers at the local level, laboratories, etc. is part of the process.

- $\quad$ Outbreak algorithms can be scheduled as needed, e.g. daily or at least weekly, as well as run on an ad hoc basis.

- Algorithms can be fine-tuned easily and can also be applied to subsets of the data.

- $\quad$ Routines are in place for assessing if follow-up of a signal is needed.

- $\quad$ Routines are well documented, including vacation replacement strategies.

- Evaluation strategy is defined and regular evaluations are scheduled.

- The system supports logging of judgements of the detected signals to allow for future analysis and improvement of the algorithms.

- System-generated alerts can be linked to reported outbreaks.

- $\quad$ Signals presented once can be suppressed by the user until a second threshold is crossed.

- Sufficient support and maintenance of the hardware and software is provided. Also routines for user support and maintenance of software and hardware are documented. 
Usability

Well-functioning computerised outbreak detection should be able to detect outbreaks of infectious diseases, to notify the people in charge of the surveillance and possibly also to reduce the workload of those working in outbreak detection. It is therefore crucial to not only improve the algorithms but to give the users the tools they need - that is, to find a suitable way to present the signals, which works for the receiver (for example, showing an epidemic curve of the cases contributing to the signal, visualising the expected maximum number or threshold, and showing a map that displays the regional distribution of the cases). The outbreak alert system should be tightly integrated with the surveillance database and allow the user easy access to the case reports that contributed to the signal. In addition, system-generated alerts can be linked to reported outbreaks. When the system is run frequently - which is critical for timely outbreak detection - users might receive the same signal several times. It is therefore important to allow the users to suppress signals presented until a second threshold is crossed. Acceptance of the system can increase if the signals can be filtered according to disease, so that those in charge of a particular disease receive signals for that disease only. Signals generated on other diseases are then not visible to them. It should be possible to schedule the algorithms as needed, for example, daily or at least weekly, as well as to run them on an ad hoc basis.

Some outbreaks affect only a certain risk group, meaning that the number of cases is so low that the excess cases cannot be detected automatically. It is therefore recommended to look at subsets of the data, such as regions, age groups and sex. Regional clustering together with time is extremely useful. In particular this may compensate for the lack of typing detail for frequently reported pathogens such as campylobacter. Changes in the system, such as new reporting laboratories, new test methods, etc., can significantly affect the performance of the algorithms and have to be carefully taken into account.

\section{Standard operating procedures}

The value of computerised outbreak detection is low if it is used only occasionally and if it is not embedded in standard operating procedures (SOPs) that clearly state the procedures for both the assessment of the signals and the actions to be taken if a detected outbreak is considered to pose a risk to the public. These SOPs should include feedback to the local level, the laboratories, etc. They should also handle more technical aspects, such as user support and maintenance of software and hardware.

\section{Evaluation}

We strongly recommend regular evaluation of the computerised outbreak detection system. This evaluation should include, for example, the system's usefulness and acceptance and should not be restricted to the performance of the algorithms used. To assess specificity, it would be helpful to log the experts' judgements of the detected signals, whether the signal indicates a real outbreak or it should be considered a false alarm. Information on outbreaks detected by more traditional means, such as by people in laboratories or at local health authorities, can be used to assess the sensitivity and timeliness of the algorithms.

Other aspects of a computerised system, such as report generation and presentation and visualisation of the data related to an outbreak, should also be evaluated.

\section{Discussion}

The different algorithms described in this paper have shown their ability to detect outbreaks that without their application would have been detected later or maybe even remained unnoticed. However, despite the obvious usefulness of the algorithms or the outbreak detection procedure itself, all countries have experienced certain limitations of the systems.

At all six institutes, the electronic systems are a central part of the outbreak detection process. The output is used in several complementary ways, and signals can often raise awareness among the people in charge of disease surveillance. The signals are either sent automatically from the system directly to a wider audience, or may already be filtered by a trained administrative clerk or an epidemiologist before being disseminated for further assessment. Tables, charts and maps, as well as results of statistical analyses by the outbreak detection algorithms are used to aid the assessment of the relevance of a signal. In addition, contextual information might be needed from other departments, for example, agricultural and census data. In several of the countries, the output of the system is also published on internal or public websites, allowing information to be shared with a broader audience as well as feedback to be given to the information provider.

By using a computerised system, it is possible to analyse data at various aggregation levels (e.g. different administrative levels) as well as data on different subsets of the population (e.g. by sex and age group). In addition, hundreds of pathogens can be analysed in a short period of time. The use of different outbreak detection methods, ranging from simple thresholds to complex statistical algorithms, in combination with the possibility of fine-tuning the system over time means that the system can be adapted according to different disease patterns. Running the algorithms on hundreds of pathogens and on different population subsets is, however, likely to pose a problem of many false alerts, which can reduce the usefulness of a computerised system. Although fine-tuning the system over time might reduce the problem, sufficient human resources are needed to deal with the generated alerts.

It has been noted by the users of the systems described that automatically detected disease clusters frequently have been observed at the same time or even earlier by 
someone else, for example, by a laboratory. In such a case, the role of the system is rather that of providing further evidence and acting as a complement to the traditional surveillance. However, several countries also report that outbreaks that would otherwise have been missed have been detected by the computerised systems (for references, see for example [9]).

We consider that following the recommendations presented in this paper is a prerequisite for an optimally functioning computer-supported outbreak detection system. In so doing, a system that is user-friendly and supports a complex epidemiological reality may be obtained.

\section{References}

1. Lawson AB, Kleinman K, editors. Spatial and syndromic surveillance for public health. London: Wiley; 2005.

2. Unkel S., Farrington CP, Garthwaite PH, Robertson C, Andrews N. Statistical methods for the prospective detection of infectious disease outbreaks: a review. Milton Keynes: Open University; 2010. Statistics Group, Technical Report 10/o8. Available from: http://stats-www.open.ac.uk/ TechnicalReports/OutbreakReviewPaper.pdf

3. Ternhag A, Tegnell A, Lesko B, Skaerlund K, Penttinen P. Basic Surveillance Network, a European database for surveillance data on infectious diseases. Euro Surveill. 2004;9(7):pii=472. Available from: http://www.eurosurveillance.org/ViewArticle. aspx?Articleld $=472$

4. Amato-Gauci A, Ammon A. ECDC to launch first report on communicable diseases epidemiology in the European Union. Euro Surveill. 2007;12(23):pii=3213. Available from: http:// www.eurosurveillance.org/ViewArticle.aspx?Articleld $=3213$

5. Farrington CP, Andrews NJ, Beale AD, Catchpole MA. A statistical algorithm for the early detection of outbreaks of infectious Disease. J R Stat Soc A. 1996;159:547-63.

6. Ethelberg S, Hanon FX, Gerner-Smidt P, Olsen KE, Wegener HC, Mølbak K. 5 years use of an outbreak detection algorithm for diarrheagenic bacteria, Denmark. In: Program and abstracts book. International Conference on Emerging Infectious Diseases 2006, Atlanta, Georgia, USA, March 19-22, 2006. Washington, DC: American Society for Microbiology; 2006. Abstract.

7. Faensen D, Claus H, Benzler J, Ammon A, Pfoch T, Breuer T, et al. SurvNet@RKI - a multistate electronic reporting system for communicable diseases. Euro Surveill. 2006;11(4):pii=614 Available from: http://www.eurosurveillance.org/ViewArticle. aspx?Articleld $=614$

8. Krause G, Altmann D, Faensen D, Porten K, Benzler J, Pfoch $T$, et al. SurvNet electronic surveillance system for infectious disease outbreaks, Germany. Emerg Infect Dis. 2007;13(10):1548-55.

9. Straetemans M, Altmann D, Eckmanns T, Krause G. Automatic outbreak detection algorithm versus electronic reporting system. Emerg Infect Dis. 2008;14(10):1610-2.

10. Stroup DF, Wharton M, Kafadar K, Dean AG. Evaluation of a method for detecting aberrations in public health surveillance data. Am J Epidemiol. 1993;137(3):373-80.

11. Schnitzler J. Prototypische Entwicklung einer Abfragemöglichkeit eines Infektionsmelderegister zur Frühwarnung bei Ausbrüchen [The development of a query interface prototype to an infectious disease register for early warning of outbreaks]. Diplomarbeit Medizinische Informatik, Technische Fachhochschule Berlin, Germany, 2003.

12. Höhle $M$. An R-package for the monitoring of infectious diseases. Comput Stat. 2007;22(4):571-82. Available from: http://www.springerlink.com/content/a230702725×33r46/

13. Kulldorff M. A spatial scan statistics. Comm Stat Theory Methods. 1997;26(6):1481-96. Available from: http://www. informaworld.com/smpp/content $\sim \mathrm{db}=$ all content=a780115529

14. Dean A, Smith J. Epi Map 2 manual. London: Brixton Books; 1996. Available from: http://www.cdc.gov/Epiinfo/Epi6/ei6. htm
15. Feil F, Dreesman J, Steffens I. Tuberculosis screening of Aussiedler at the Friedland border immigration centre, Germany. Euro Surveill. 2004;8(18): $\mathrm{pii}=2457$. Available from: http://www.eurosurveillance.org/ViewArticle. aspx?Articleld $=2457$

16. Dreesman J, Villarroel-Conzales DC, Cleves S, Reins HA, Pulz M. [Regionally increased incidence of notified cryptosporidiosis cases due to different laboratory methods].

17. Gesundheitswesen. 2007;69(8-9):483-7. German. Widdowson MA, Bosman A, van Straten E, Tinga M, Chaves S, van Eerden $\mathrm{K}$, et al. Automated, laboratory-based system using the Internet for disease outbreak detection, the Netherlands. Emerg Infect Dis. 2003;9(9):1046-52.

18. Rahamat-Langendoen JC, Vliet van JA, Suijkerbuijk AWM Recognition of threats caused by infectious diseases in the Netherlands: the early warning committee. Eurosurveillance, 2006; 11(12):pii=672. Available from: http://www. eurosurveillance.org/ViewArticle.aspx?Articleld $=672$

19. van Pelt W, Mevius DJ, Stoelhorst HG, Kovats S, van de Giessen AW, Wannet WJ, et al. A large increase of Salmonella infections in 2003 in the Netherlands: hot summer or side effect of the avian influenza outbreak? Euro Surveill. 2004;9(7): pii=473. Available from: http://www.eurosurveillance.org/ViewArticle. aspx?Articleld $=473$

20. Cakici B, Hebing K, Grünewald $M$, Saretok P, Hulth A. CASE - a framework for computer supported outbreak detection. BMC Med Inform Decis Mak. 2010;10:14.

21. GNU Operating System. GNU General Public License. Free Software Foundation; [Accessed 27 Aug 2010]. Available from: http://www.gnu.org/licenses/gpl.html

22. Kulldorff M, Heffernan R, Hartman J, Assunção R, Mostashari F. A space-time permutation scan statistic for disease outbreak detection. PLoS Med. 2005;2(3):e59.

23. Frisén M, Andersson E, Schiöler L. Robust outbreak surveillance of epidemics in Sweden. Stat Med. 2009;28(3):476-93. 